University of Nebraska - Lincoln

DigitalCommons@University of Nebraska - Lincoln

Publications from USDA-ARS / UNL Faculty

U.S. Department of Agriculture: Agricultural

Research Service, Lincoln, Nebraska

2017

Noise-Resistant Spectral Features for Retrieving Foliar Chemical

Parameters

Jingcheng Zhang

Hangzhou Dianzi University, zhang_rs@163.com

Yanbo Huang

USDA-ARS, yanbo.huang@ars.usda.gov

Zhenhai Li

Beijing Research Center for Information Technology in Agriculture, zhuifeng3117@126.com

Peng Liu

Hangzhou Dianzi University

Lin Yuan

Zhejiang University ofWater Resources and Electric Power, y16690@126.com

Follow this and additional works at: https://digitalcommons.unl.edu/usdaarsfacpub

Zhang, Jingcheng; Huang, Yanbo; Li, Zhenhai; Liu, Peng; and Yuan, Lin, "Noise-Resistant Spectral Features for Retrieving Foliar Chemical Parameters" (2017). Publications from USDA-ARS / UNL Faculty. 2077. https://digitalcommons.unl.edu/usdaarsfacpub/2077

This Article is brought to you for free and open access by the U.S. Department of Agriculture: Agricultural Research Service, Lincoln, Nebraska at DigitalCommons@University of Nebraska - Lincoln. It has been accepted for inclusion in Publications from USDA-ARS / UNL Faculty by an authorized administrator of DigitalCommons@University of Nebraska - Lincoln. 


\title{
Noise-Resistant Spectral Features for Retrieving Foliar Chemical Parameters
}

\author{
Jingcheng Zhang ${ }^{(1)}$, Yanbo Huang ${ }^{(0)}$, Zhenhai Li, Peng Liu, and Lin Yuan
}

\begin{abstract}
Foliar chemical constituents are important indicators for understanding vegetation growing status and ecosystem functionality. Provided the noncontact and nondestructive traits, the hyperspectral analysis is a superior and efficient method for deriving these parameters. In practice, thespectral noise issue significantly impacts the performance of the hyperspectral retrieving system. To systematically investigate this issue, by introducing varying levels of noise to spectral signals, an assessment on noiseresistant capability of spectral features and models for retrieving concentrations of chlorophyll, carotenoids, and leaf water content was conducted. Given the continuous waveletanalysis (CWA) showed superior performance in extracting critical information associating plants biophysical and biochemical status in recent years, both wavelet features (WFs) and some conventional features (CFs) were chosen for the test. Two datasets including a leaf optical properties experiment dataset $(n=330)$, and a corn leaf spectral experiment dataset $(n=213)$ were used for analysis and modeling. The results suggested that the WFs had stronger correlations with all leaf chemical parameters than the CFs. According to an evaluation by decay rate of retrieving error that indicates noise-resistant capability, both WFs and CFs exhibited strong resistance to spectral noise. Particularly for WFs, the noise-resistant capability is relevant to the scale of the features. Based on the identified spectral features, both univariate and multivariate retrieving models were established and achieved satisfactory accuracies. Synthesizing the retrieving accuracy, noise resistivity, and model's complexity, the optimal univariate WFmodels were recommended in practicefor retrieving leaf chemical parameters.
\end{abstract}

Index Terms-Noise, parameter estimation, vegetation, wavelet transforms.

Manuscript received October 10, 2016; revised February 10, 2017 and May 5, 2017; accepted May 28, 2017. Date of publication June 19, 2017; date of current version December 19, 2017. This work was supported in part by the National Natural Science Foundation of China under Grant 41671415 and Grant 41601461, in part by Zhejiang Public Welfare Programme of Agriculture Technology under Grant 2016C32087, and in part by Beijing Nova Programme under Grant Z151100000315059, China. (Corresponding author: Yanbo Huang.)

J. Zhang and P. Liu are with the College of Life Information Science and Instrument Engineering, Hangzhou Dianzi University, Hangzhou 310018, China (e-mail: zhang_rs@163.com; 1016561228@qq.com).

Y. Huang is with the Crop Production Systems Research Unit, United States Department of Agriculture, Agricultural Research Service, Stoneville, MS 38776 USA (e-mail: yanbo.huang@ars.usda.gov).

$\mathrm{Z}$. $\mathrm{Li}$ is with Beijing Research Center for Information Technology in Agriculture, Beijing 100097, China (e-mail: zhuifeng3117@126.com).

L. Yuan is with the School of Information Engineering and Art and Design, Zhejiang University of Water Resources and Electric Power, Hangzhou 310018, China (e-mail: y16690@126.com).

Color versions of one or more of the figures in this paper are available online at http://ieeexplore.ieee.org.

Digital Object Identifier 10.1109/JSTARS.2017.2713039

\section{INTRODUCTION}

$\mathbf{F}$ OLIAR chemical constituents (e.g., pigments, water, lignin, cellulose, nitrogen) are critical indicators for understanding vegetation productivity, nutrient status, stressors, and other functional processes at ecosystem level [1]. To obtain foliar chemical contents nondestructively, hyperspectral analyses are the mainstream methods allowing noncontact measurement in real time [2]-[4].

The interaction of electromagnetic radiation with plant leaves (reflection, transmission, absorption) was governed by their chemical constitution and physical characteristics. Variation of these chemical constituents (e.g., chlorophyll, water, etc.) would result in specific changes on reflectance over the visible (VIS) and near infrared (NIR) regions (0.4-2.4 $\mu \mathrm{m}$ ), which serves as a physical basis for establishing their retrieval models. In previous studies, many vegetation indices (VIs) that apply algebraic combination on specific spectral bands were proposed and linked with chemical constituents. For example, Kim et al. [5] developed the chlorophyll absorption in reflectance index (CARI) to estimate foliar chlorophyll (Chl) content. The index was advanced in minimizing the effects of nonphotosynthetic materials. In estimating foliar carotenoid (Car) content, Gitelson et al. [6] proposed the Car reflectance index (CRI) that uses a reciprocal reflectance at either 550 or $700 \mathrm{~nm}$ to remove the $\mathrm{Chl}$ effect. Besides, as mentioned by Gitelson et al. [7], the tuning of spectral regions was important in retrieving foliar pigments content (i.e., Chls, Cars, and anthocyanins). Given the significance of pigments in plant functions, some researchers pointed out that links between foliar pigment contents and some important plant physiological parameters (i.e., leaf nitrogen, green leaf area index, gross primary production) could also be established [8], [9]. Besides the pigments content, the retrieving of foliar water content is another important task for understanding the growing status of the plant. Based on the near- and middle-infrared reflectances, Hunt and Rock [10] proposed a moisture stress index (MSI) to detect foliar water content. In addition, a simple ratio of R900/R970 was developed to estimate plants' water content [11]. Besides the VIs, derivative spectral features and continuum-removal features (Der \& Con features) were noiseresistant features that were used for retrieving foliar biochemical parameters. The derivative spectral features are able to remove or mitigate some noise effects such as illumination variations caused by terrain background, atmosphere, viewing geometry [12]. The continuum-removal features are efficient spectral analytical tool to extract spectral absorption features including

1939-1404 @ 2017 IEEE. Personal use is permitted, but republication/redistribution requires IEEE permission. See http://www.ieee.org/publications_standards/publications/rights/index.html for more information. 
absorption band depth, width, position, area, and asymmetry [13]. The retrieving results of foliar biochemical parameters by derivative or continuum-removal spectral features are usually better than using band reflectance. Asner and Martin [2] found that the derivative features can be well correlated with foliar pigments (Chls and Cars). Pu et al. [14] reported the effectiveness of the continuum-removal features in retrieving leaf relative water content of oak trees.

Apart from the VIs and Der \& Con features, it is noticed that a signal processing method, the continuous wavelet analysis (CWA), emerged as a promising tool in identifying efficient spectral features for estimating foliar leaf chemical constituents [15], [16]. The CWA-derived wavelet features (WFs) were successfully correlated with foliar water content and leaf mass [17], [18]. Using airborne hyperspectral image data, Cheng et al. [19], [20] showed that the WFs also held the potential on retrieving canopy-level water content under relatively high noise circumstance. Moreover, given many plant stresses would lead to variation of plants' leaf chemical constituents, the spectral response of the stressed plants would be thus altered. In this case, the CWA method was also proven to be an efficient tool in identifying WFs for detecting plant diseases and pests, freezing damage, etc. [21]-[23].

In practice, the noise interference is a common issue to applications of hyperspectral data [24], [25]. Although the spectral smoothing methods can deal with some extent of noise interference, the information loss might occur at the same time. Therefore, the ideal and robust spectral features for retrieving chemical constituents are expected to have not only high sensitivity to the retrieved parameter, but also strong tolerance to the spectral noise. However, the noise-resistant capability for both VIs and CWA-derived WFs remains unclear. To investigate this issue, by introducing different levels of noise to spectral signals, a systematic check on noise-resistant capability was performed for both conventional features (CFs) and WFs. It is expected to identify the most efficient and robust spectral features for retrieving critical foliar chemical parameters, including two foliar pigments: Chl, Car; and a leaf water content (LWC). Therefore, the objectives of this study are:

1) to compare the sensitivity to foliar chemical parameters between CFs and CWA-derived WFs;

2) to assess and compare the noise-resistant capability between the two types of features; and

3) to develop retrieving models of foliar chemical parameters based on the most robust spectral features.

\section{MATERIALS AND METHODS}

The entire analytical workflow was illustrated in Fig. 1. In this study, two leaf-level spectral datasets that are relevant to foliar chemical parameters and corresponding spectral measurements were applied to perform a CWA to identify the most efficient WFs. The sensitivity to foliar chemical parameters of the WFs and CFs was assessed and compared. Besides, by introducing different levels of noise to spectral data, the noise-resistant capability was analyzed for the CFs and WFs, which helps to identify the most robust features for retrieving foliar chemical parameters. Based on these features with the univariate and multivariate

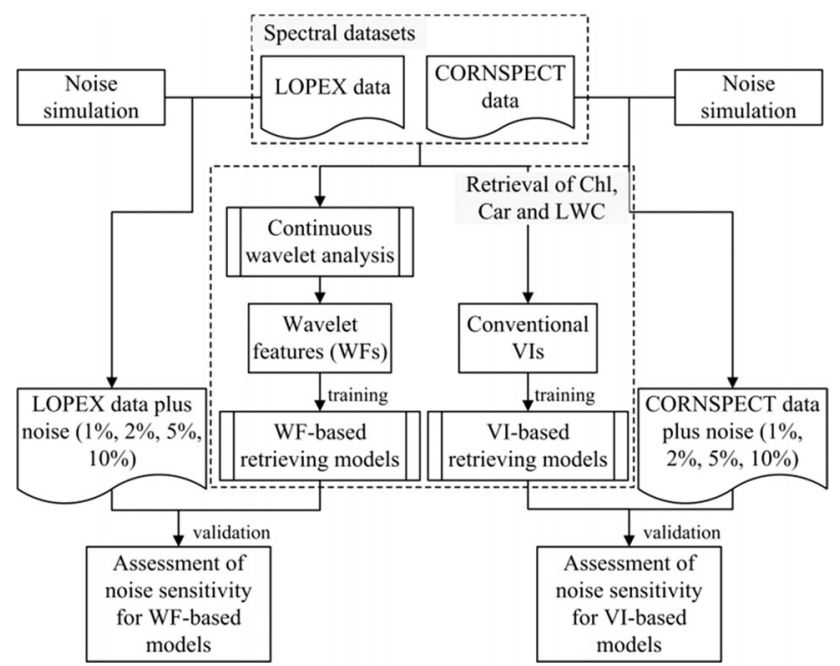

Fig. 1. Flowchart of the feature selection and modeling process for retrieving leaf chemical parameters.

regression methods, the optimal retrieving models that have the highest accuracy and strongest noise-resistant capability were determined for Chl, Car, and LWC.

\section{A. Dataset Description}

The leaf optical properties experiment (LOPEX) data and the corn leaf spectral experiment (CORNSPEC) data are relevant to water content and pigments content (i.e., both $\mathrm{Chl}$ and Car), respectively. For analyzing the relationship between concentrations of corns' major photosynthetic pigments and spectral signals, the CORNSPEC dataset was collected in 2013 at Beijing Xiaotangshan Precision Agriculture Experimental Base, Beijing, China. The dataset included a total of 213 corn leaf samples being measured for both spectral reflectance and concentrations of Chl and Car on July 31th, August 14th, and August 28th, corresponding to the growing period from the jointing stage to the end of the silking stage. The leaf spectra were measured by a FieldSpec UV/VNIR spectroradiometer (ASD Inc., Boulder, Colorado, USA) over 400-2500 nm wavelengths, coupled with an ASD Leaf Clip. A total of ten readings were recorded and averaged to obtain a spectral measurement for each leaf. The spectrum of a white Spectralon reference panel (99\% reflectance) was measured once for every ten leaf measurements. Leaf reflectance was calculated by dividing the sample radiance with the radiance of the reference panel. Right after the spectral measurements, the exact portion of leaf that received spectral measurement was cut and placed in a tube with $10 \mathrm{ml}$ dimethyl sulfoxide. The pigments were extracted by placing the tube in a $65{ }^{\circ} \mathrm{C}$ water tub in a dark room for more than $5 \mathrm{~h}$. Then, the chlorophylla ( $\mathrm{Chl} a$ ), chlorophyllb (Chlb), and Cars were extracted and their concentrations were computed using the equations of Lichtenthaler et al. [26] as follows:

$$
\begin{aligned}
& C_{A}=12.25 \mathrm{OD}_{663}-2.79 \mathrm{OD}_{647} \\
& C_{B}=21.50 \mathrm{OD}_{647}-5.10 \mathrm{OD}_{663} \\
& C_{C}=\left(1000 \mathrm{OD}_{470}-1.82 C_{A}-85.02 C_{B}\right) / 198
\end{aligned}
$$


TABLE I

INFORMATION OF DATASETS USED IN THIS STUDY

\begin{tabular}{|c|c|c|c|c|}
\hline \multirow[b]{2}{*}{ Dataset } & \multirow[b]{2}{*}{ Sample size } & \multirow[b]{2}{*}{ Parameters } & \multicolumn{2}{|c|}{ Basic statistics } \\
\hline & & & Mean & SD \\
\hline LOPEX93 (Mixture of 42 plants) & $\begin{array}{c}\text { Calibration }(n=198) \text {; } \\
\text { Validation }(n=132)\end{array}$ & LWC & $66.08 \%$ & $12.16 \%$ \\
\hline CORNSPEC (Maize) & $\begin{array}{c}\text { Calibration }(n=129) \\
\text { Validation }(n=84)\end{array}$ & $\begin{array}{l}\operatorname{Chl}\left(\mu \mathrm{g} / \mathrm{cm}^{2}\right) \\
\operatorname{Car}\left(\mu \mathrm{g} / \mathrm{cm}^{2}\right)\end{array}$ & $\begin{array}{c}49.57 \\
8.97\end{array}$ & $\begin{array}{l}9.24 \\
1.45\end{array}$ \\
\hline
\end{tabular}

where $C_{A}, C_{B}$, and $C_{C}$ are concentrations of $\mathrm{Chl} a, \mathrm{Chl} b$, and Car in $\mathrm{mg} \cdot \mathrm{L}^{-1}$, respectively. $\mathrm{OD}_{447}, \mathrm{OD}_{670}$, and $\mathrm{OD}_{663}$ are the absorbency at specific wavelengths. Hereafter, the concentrations of Chla $+\mathrm{Chl} b$ and Car are represented by Chl and Car, respectively.

While for analysis on foliar water content, a commonly used dataset, the LOPEX data, was applied in this study. The LOPEX data were collected by the Joint Research Centre in Italy for exploring relationships between foliar chemical constituents and spectral signals [27]. The dataset was widely used in remote sensing community for feature selection and model calibration/validation [17], [28], [29]. From the dataset, the portion that was relevant to LWC was taken to conduct the analysis, which included a total of 330 measurement of spectral reflectance over the range of 400-2500 $\mathrm{nm}$ and corresponding LWC across 45 plant species. The LWC was determined by

$$
\mathrm{LWC}_{F}=\frac{\mathrm{FW}-\mathrm{DW}}{\mathrm{FW}}
$$

where FW and DW are the fresh weight and dry weight of a leaf, respectively. Some information and basic statistics of leaf chemical parameters (i.e., mean and standard deviation) for both datasets are summarized in Table I. The dataset was randomly divided into a calibration subset $(60 \%)$ and a validation subset (40\%) to allow an independent model validation.

\section{B. Spectral Noise}

To systematically evaluate the noise-resistant capability of wavelet and CFs, varying degrees of stochastic noise were introduced to the spectra in LOPEX and CORNSPEC datasets. The noise was generated conforming Gaussian distribution across samples, with mean of zero and standard deviation as a proportion of the variance of the original spectra. Four levels of noise were generated with proportion of $1 \%, 2 \%, 5 \%$, and $10 \%$. The noise was added to the original spectra to form the noiseinterfered spectra for further tests.

\section{CWA Method for Feature Selection}

As a tool in signal processing, wavelet analysis has been successfully applied in processing the hyperspectral data for dimensionality reduction and noise suppression [30]-[32]. Different from the conventional VIs that mostly rely on the reflectance magnitude at some specific bands, the CWA is capable in decomposing a signal at continuous scales and wavelengths, which permits a traversal of the entire spectra to search for the most sensitive spectral features. Besides, since the CWA-derived features are directly comparable to the original spectra, it is possible to readily interpret the features by comparing its shape and central wavelength with the spectral absorption regions [16], [33]. In light of the merits of CWA in information extraction, the method is used for identifying the most sensitive WFs for retrieving leaf chemical parameters. Fig. 1 demonstrates the CWA procedure for spectral feature selection, which includes the implementation of continuous wavelet transform, and the identification of WFs.

1) Implementation of Continuous Wavelet Transform: First, based on a mother wavelet basis function, a wavelet transformation was implemented on each of the spectrum, to convert the original reflectance spectrum to a set of coefficients over continuous wavelengths and scales. The transformation operates the scaling and shifting process to the mother wavelet $\Psi(\lambda)$ following the equation:

$$
\Psi_{a, b}(\lambda)=\frac{1}{\sqrt{a}} \Psi\left(\frac{\lambda-b}{a}\right)
$$

where $a$ is the scaling factor indicating the width of the wavelet, and $b$ is the shifting factor indicating the position [34]. In this way, the spectrum is converted to a set of power coefficients, as indicated by the following equation:

$$
W_{f}(a, b)=f, \Psi_{a, b}=\int_{-\infty}^{+\infty} f(\lambda) \Psi_{a, b}(\lambda) d \lambda
$$

where $f(\lambda) \quad(\lambda=1,2, \ldots n, n$ is the number of wavebands, in this study $n=2500-400+1=2101$ with the spectral range from 400 to $2500 \mathrm{~nm}$ with $1 \mathrm{~nm}$ interval) represents the spectrum. The coefficients $\left(W_{f}\left(a_{i}, b_{j}\right), i=\right.$ $1,2, \ldots, m, j=1,2, \ldots, n)$ constitute a scalogram (an $m$ $\times n$ matrix), with scale and wavelength serving as two dimensions. As for the type of mother wavelet, the second derivative of Gaussian function (also known as the Mexican hat) was chosen as the mother wavelet functions with two considerations: first, given the shape of the vegetation absorption features approached to Gaussian or quasi-Gaussian function, Mexican hat function facilitates capturing these features. Moreover, as a convolutional filter, the Mexican hat function has a superior trait in concentrating energy for transformed signal and was proven to have denoise feature in spectral analysis [20], [23], [35], [36]. Given strong correlation existed among wavelet powers at adjacent scales, only data at dyadic scales as $2^{1}, 2^{2}, 2^{3}, \ldots$, and $2^{10}$ were sufficient to characterize the spectral response, and were thus retained in the scalogram, so as to reduce the computational load of the analysis [19].

2) Identification of Wavelet Features: To relate the derived scalogram with leaf chemical parameters, a linear correlation analysis was employed to generate coefficients of determination $\left(R^{2}\right)$ between wavelet coefficients and each leaf chemical parameter across all samples. Therefore, the corresponding $R^{2}$ values will constitute a correlation scalogram (also an $m \times n$ matrix), which is used for indicating the sensitivity of each wavelet coefficient to leaf chemical parameter.

Based on the correlation scalogram, the most sensitive WFs were identified according to a thresholding method [17], [23]. To achieve this goal, all WFs were sorted in a descending order of $R^{2}$, and a cut-off value of $R^{2}$ was applied to retain $1 \%$ elements that showed the strongest correlation with the targeted 
parameter. This process will result in a number of feature regions in the scalogram. To facilitate the calculation of WFs, in each feature region, only the element with the highest $R^{2}$ was chosen, which not only guaranteed to find the features that were the most sensitive to the leaf chemical parameters, but also facilitated the calculation of the feature. For each parameter, corresponding WFs were identified using the original spectral data (noise-free). These WFs with fixed central wavelength and scale were then used on the noise-added datasets.

\section{Conventional Spectral Features}

Apart from WFs, two types of conventional spectral features were also tested and compared with WFs in retrieving leaf chemical parameters, including a set of classic VIs and a set of derivative and continuum-removal spectral features (Der $\&$ Con features). According to a thorough literature review, a number of published VIs were selected to be correlated with leaf chemical parameters. All these selected VIs were well documented and recommended for monitoring bioparameters [12]. For retrieving water content, five VIs were selected, including leaf water VI (LWVI-1, LWVI-2), MSI, normalized difference water index (NDWI), and water index (WI). For the two pigment attributes, pigment-specific simple ratio (PSSR), CRI, and double difference (DD) were used to retrieve Chl, and modified normalized difference (mND705, mND680), triangular VI (TVI), CARI, and modified CARI (MCARI) were used to retrieve Car. Besides, 15 classic Der \& Con features that capture some important spectral absorption regions (e.g., blue edge, yellow edge, red edge) of vegetation were selected to be associated with Chl, Car, and LWC, respectively. The expressions and descriptions of the aforementioned VIs and Der \& Con features are listed in Tables II and III. Similar to the CWA procedure, a correlation analysis was performed to determine the sensitivity of these VIs and Der \& Con features to corresponding leaf chemical parameters.

\section{E. Noise Sensitivity Analysis and Retrieving Model}

The noise sensitivity of spectral features was quantified according to a decay rate of the retrieving accuracy with increase of noise levels. The root mean square error (RMSE) was used to reflect the retrieving accuracy, which was calculated based on univariate linear regression model. For each spectral feature (i.e., wavelet or $\mathrm{CF}$ ), the model was trained by the calibration data, and the RMSE was calculated using the validation data:

$$
\mathrm{RMSE}=\sqrt{\frac{\sum_{i=1}^{n}\left(y_{\mathrm{est}, i}-y_{\mathrm{obs}, i}\right)^{2}}{n}}
$$

where $n$ is the sample size; $y_{\text {est }}$ is the model estimate of the parameter; and $y_{\mathrm{obs}}$ is the measured value of the parameter. Based on the RMSE of the models that were calibrated by noise-free data and noise-added data at various levels, the decay rate (denotes as DecayRate hereafter) can be calculated by

$$
\begin{aligned}
& \text { DecayRate }= \\
& \times\left[\left(\mathrm{RMSE}_{\text {maximal }}-\mathrm{RMSE}_{\text {normal }}\right) / \mathrm{RMSE}_{\text {normal }}\right]-100 \%
\end{aligned}
$$

where $\mathrm{RMSE}_{\text {maximal }}$ is the maximal RMSE (indicating the lowest retrieving accuracy) among the models calibrated with noise-added data at different levels; $\mathrm{RMSE}_{\text {normal }}$ is the RMSE corresponding to the model calibrated with noise-free data.

To establish retrieving models for leaf chemical parameters, a feature selection procedure was conducted to consider the sensitivity of features and the features' noise-resistant capability. Therefore, the first criterion is that the feature requires a strong correlation with the targeted parameter. Here, a strict rule on the correlation analysis with confidence level of $99.9 \%$ ( $p$-value $<0.001$ ) was used to ensure that the selected spectral features have sufficient sensitivity to leaf chemical parameters. The second criterion is that the feature needs to have a relatively low DecayRate. Through many trials, an upper threshold of 0.2 was determined for the DecayRate to ensure that the selected spectral features have a strong capability of noise resistance.

Based on the above-mentioned criteria, the selected spectral features served as input variables for retrieving models. Both the linear regression univariate model and partial least squares regression (PLSR) multivariate model were constructed for each retrieving leaf chemical parameters. All the models were trained with the calibration dataset and assessed with the validation datasets. To evaluate and compare the performance of different models, the coefficient of determination $\left(R^{2}\right)$ and RMSE were used as accuracy measures. All statistical analyses were conducted with the programs of the MATLAB software (MathWorks Inc., Natick, MA, USA).

\section{RESULTS}

\section{A. Responses of Wavelet and Conventional Features to Leaf Chemical Parameters}

As shown in Fig. 2, a gradient of Chl, Car, and LWC would induce corresponding spectral responses that mainly occurred over different spectral regions. The variation of $\mathrm{Chl}$ and $\mathrm{Car}$ contents mainly caused spectral variation in VIS and NIR regions, whereas variation of LWC would induce spectral changes in NIR and short wave infrared (SWIR) regions. Such pattern provides a basis of retrieving leaf chemical parameters with spectral measurements.

To identify the most sensitive WFs to the leaf chemical parameters, the CWA and correlation analysis were performed, which yielded three separate correlation scalograms indicating correlation across different wavelengths and scales (see Fig. 3). According to the features selection criteria as described in Section II-C, the most sensitive WFs were identified for the three leaf chemical parameters (see Table IV). For Chl, five WFs with $R^{2}$ ranging from 0.79 to 0.82 were identified. While for Car, only two WFs with $R^{2}$ around 0.65 were identified. Comparing with the pigments, more WFs $(n=7)$ were identified for LWC, with $R^{2}$ ranging from 0.73 to 0.86 . The central wavelength and scale of the WFs provided their characteristics. For Chl, the central wavelength of the WFs located in green, red, and NIR spectral regions (i.e., 550-900 nm), with their scale spanning from low (scale $=2$ ) to high (scale $=6$ ). For Car, both the WFs located at relatively high scale (scale $=6$ ) in NIR region, with their central 
TABLE II

LisT OF CONVENTIONAL VEGETATION INDICES USED IN THIS STUDY

\begin{tabular}{|c|c|c|c|}
\hline $\begin{array}{l}\text { Leaf chemical } \\
\text { parameters }\end{array}$ & $\begin{array}{l}\text { Vegetation } \\
\text { indices (VIs) }\end{array}$ & Expression & Publication \\
\hline \multirow[t]{5}{*}{ Water } & LWVI-1 & $(\mathrm{R} 1094-\mathrm{R} 893) /(\mathrm{R} 1094$ + R893) & Galvão et al., 2005 \\
\hline & LWVI-2 & $(\mathrm{R} 1094-\mathrm{R} 1205) /(\mathrm{R} 1094+\mathrm{R} 1205)$ & Galvão et al., 2005 \\
\hline & MSI & $\mathrm{R} 1600 / \mathrm{R} 819$ & Hunt and Rock, 1989; Ceccato et al., 2001 \\
\hline & NDWI & $(\mathrm{R} 860-\mathrm{R} 1240) /(\mathrm{R} 860+\mathrm{R} 1240)$ & Datt et al., 2003; Gao, 1996 \\
\hline & WI & R900/R970 & Pe elas et al., 1997 \\
\hline \multirow[t]{3}{*}{ Car } & PSSR & $\mathrm{R} 800 / \mathrm{R} 500$ & Blackburn, 1998 \\
\hline & CRI & $\begin{aligned} \text { CRI550 }=(\mathrm{R} 510) & -1-(\mathrm{R} 550)-1, \mathrm{CRI} 700=(\mathrm{R} 510) \\
& -1-(\mathrm{R} 700)-1\end{aligned}$ & Gitelson et al., 2002 \\
\hline & DD & $(\mathrm{R} 750-\mathrm{R} 720)-(\mathrm{R} 700-\mathrm{R} 670)$ & le Maire et al., 2004 \\
\hline \multirow[t]{5}{*}{ Chl } & mND705 & $(\mathrm{R} 750-\mathrm{R} 705) /(\mathrm{R} 750+\mathrm{R} 705-2 \mathrm{R} 445)$ & Sims and Gamon, 2002 \\
\hline & TVI & $0.5[120(\mathrm{R} 750-\mathrm{R} 550)-200(\mathrm{R} 670-\mathrm{R} 550)]$ & Broge and Leblanc, 2000; Haboudane et al., 2004 \\
\hline & MCARI & {$[(\mathrm{R} 701-\mathrm{R} 671)-0.2(\mathrm{R} 701-\mathrm{R} 549)] /(\mathrm{R} 701 / \mathrm{R} 671)$} & Daughtry et al., 2000 \\
\hline & mND680 & $(\mathrm{R} 800-\mathrm{R} 680) /(\mathrm{R} 800+\mathrm{R} 680-2 \mathrm{R} 445)$ & Sims and Gamon, 2002 \\
\hline & CARI & $\begin{array}{c}(|(\mathrm{a} 670+\mathrm{R} 670+b)| /(a 2+1) 1 / 2) \times(\mathrm{R} 700 / \mathrm{R} 670) ; a= \\
(\mathrm{R} 700-\mathrm{R} 550) / 150, b=\mathrm{R} 550-(a \times 550)\end{array}$ & Kim et al., 1994 \\
\hline
\end{tabular}

TABLE III

Derivative and ContinuUm-Removal Transformed Spectral Features Used IN This STUdy

\begin{tabular}{|c|c|c|c|c|}
\hline Variable & Definition & Description & $\begin{array}{l}\text { Related } \\
\text { parameter }\end{array}$ & Literatures \\
\hline \multicolumn{5}{|c|}{ Derivative transformed spectral variables } \\
\hline $\mathrm{Db}$ & $\begin{array}{l}\text { Maximum value of first } \\
\text { derivative within blue edge }\end{array}$ & $\begin{array}{l}\text { Blue edge covers } 490-530 \mathrm{~nm} \text {. Db is a maximum value of first-order derivatives within } \\
\text { the blue edge of } 35 \text { bands }\end{array}$ & Chl, Car & Gong et al., 2002 \\
\hline $\mathrm{SDb}$ & $\begin{array}{l}\text { Sum of first derivative values } \\
\text { within blue edge }\end{array}$ & Defined by sum of first-order derivative values of 35 bands within the blue edge & Chl, Car & Gong et al., 2002 \\
\hline Dy & $\begin{array}{l}\text { Maximum value of first } \\
\text { derivative within yellow edge }\end{array}$ & $\begin{array}{l}\text { Yellow edge covers } 550-582 \mathrm{~nm} \text {. Dy is a maximum value of first-order derivatives } \\
\text { within the yellow edge of } 28 \text { bands }\end{array}$ & Chl, Car & Gong et al., 2002 \\
\hline SDy & $\begin{array}{l}\text { Sum of first derivative values } \\
\text { within yellow edge }\end{array}$ & Defined by sum of first-order derivative values of 28 bands within the yellow edge & Chl, Car & Gong et al., 2002 \\
\hline Dr & $\begin{array}{l}\text { Maximum value of first } \\
\text { derivative within red edge }\end{array}$ & $\begin{array}{l}\text { Red edge covers } 670-737 \mathrm{~nm} \text {. Dr is a maximum value of first-order derivatives within } \\
\text { the red edge of } 61 \text { bands }\end{array}$ & Chl, Car & Gong et al., 2002 \\
\hline SDr & $\begin{array}{l}\text { Sum of first derivative values } \\
\text { within red edge }\end{array}$ & Defined by sum of first-order derivative values of 61 bands within the red edge & Chl, Car & Gong et al., 2002 \\
\hline \multicolumn{5}{|c|}{ Continuum-removal transformed spectral features } \\
\hline DEP-1 & $\begin{array}{l}\text { The depth of the feature } \\
\text { minimum relative to the hull }\end{array}$ & In the range of $550-750 \mathrm{~nm}$ & Chl, Car & $\begin{array}{l}\text { Pu et al., 2003, } \\
2004\end{array}$ \\
\hline DEP-2 & & In the range of $920-1120 \mathrm{~nm}$ & LWC & \\
\hline DEP-3 & & In the range of $1070-1320 \mathrm{~nm}$ & LWC & \\
\hline WID-1 & $\begin{array}{l}\text { The full wavelength width at } \\
\text { half DEP }(\mathrm{nm})\end{array}$ & In the range of $550-750 \mathrm{~nm}$ & Chl, Car & $\begin{array}{l}\text { Pu et al., 2003, } \\
2004\end{array}$ \\
\hline WID-2 & & In the range of $920-1120 \mathrm{~nm}$ & LWC & \\
\hline WID-3 & & In the range of $1070-1320 \mathrm{~nm}$ & LWC & \\
\hline AREA-1 & $\begin{array}{l}\text { The area of the absorption } \\
\text { feature that is the product of } \\
\text { DEP and WID }\end{array}$ & In the range of $550-750 \mathrm{~nm}$ & Chl, Car & $\begin{array}{c}\text { Pu et al., 2003, } \\
2004\end{array}$ \\
\hline AREA-2 & & In the range of $920-1120 \mathrm{~nm}$ & LWC & \\
\hline AREA-3 & & In the range of $1070-1320 \mathrm{~nm}$ & LWC & \\
\hline
\end{tabular}

wavelength located at 709 and $852 \mathrm{~nm}$. For LWC, all sensitive WFs located beyond $1200 \mathrm{~nm}$ (i.e., 1200-2400 nm), with their scale ranging from 4 to 8 . By superimposing the WFs over the original spectra, it is clear that the identified WFs are able to capture the absorption features caused by variations of $\mathrm{Chl}$, Car, and LWC (see Fig. 4). Therefore, those WFs were recognized as potential input variables for retrieving leaf chemical parameters. Comparing with these WFs, the CFs showed remarkable weaker responses to all the leaf chemical parameters. Among the VIs and Der \& Con features, Db, DD, and WI exhibited the strongest correlations with $\mathrm{Chl}\left(R^{2}=0.72\right)$, Car
$\left(R^{2}=0.56\right)$, and LWC $\left(R^{2}=0.45\right)$, respectively. It is noted that some of the CFs failed to show a statistically significant correlation with the leaf chemical parameters, including mND680, AREA-1, Dr, and SDr for Chl; PSSR, CRI550, CRI700, DEP-1, AREA-1, Dr, and SDr for Car; and WID-2 and WID-3 for LWC. The CFs that passed the sensitivity analysis were also used to construct retrieving models.

\section{B. Evaluation of Noise Resistance of Spectral Features}

In retrieving leaf chemical parameters, ideal spectral features are expected to have not only sensitivity to these parameters, 


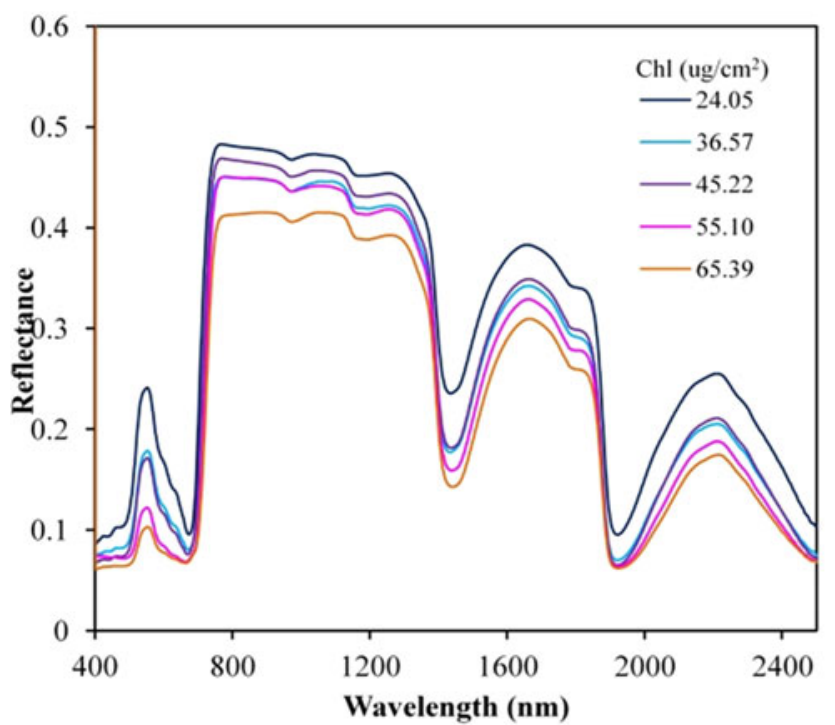

(a)

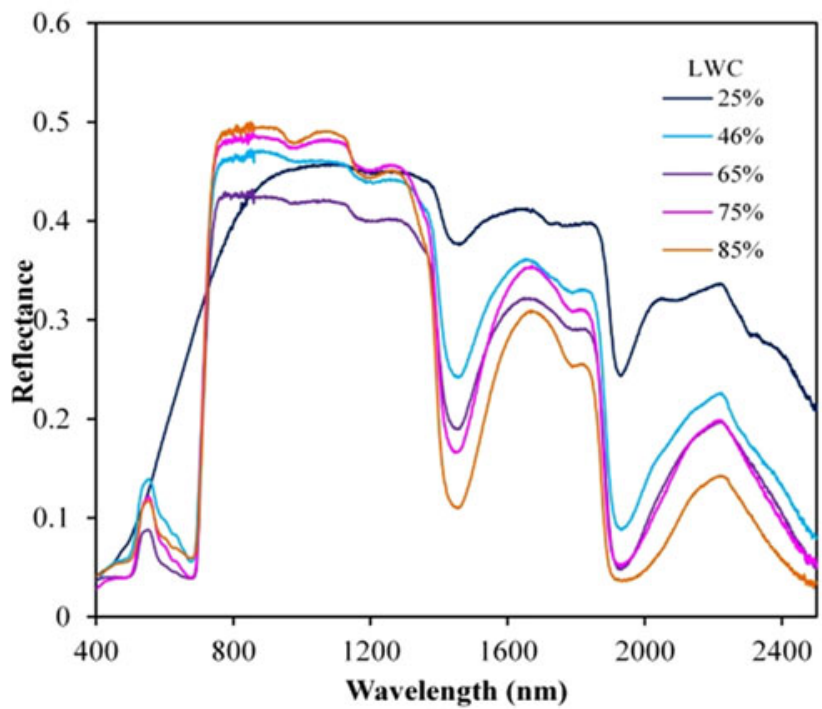

(c)

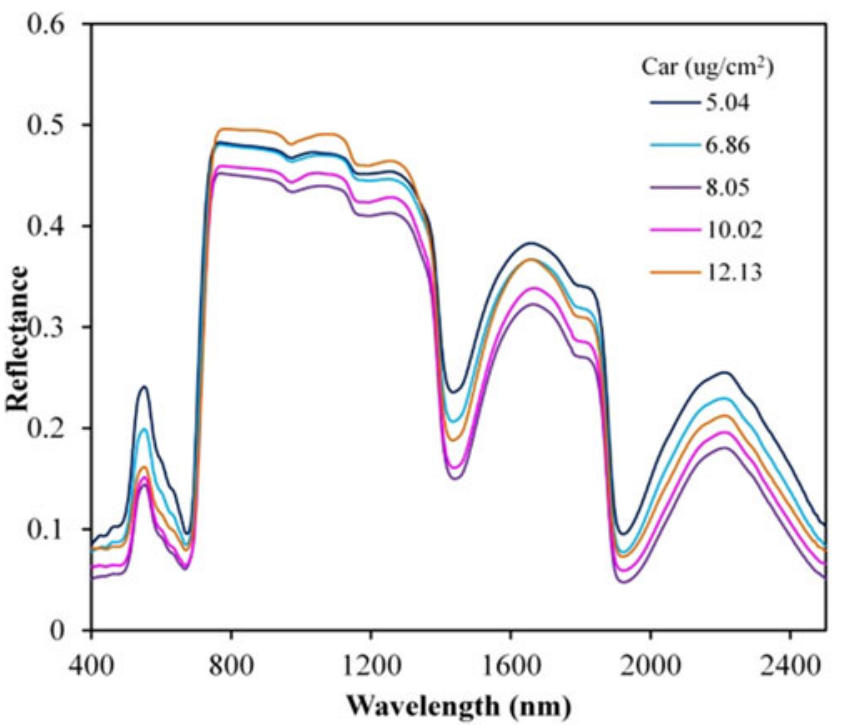

(b)

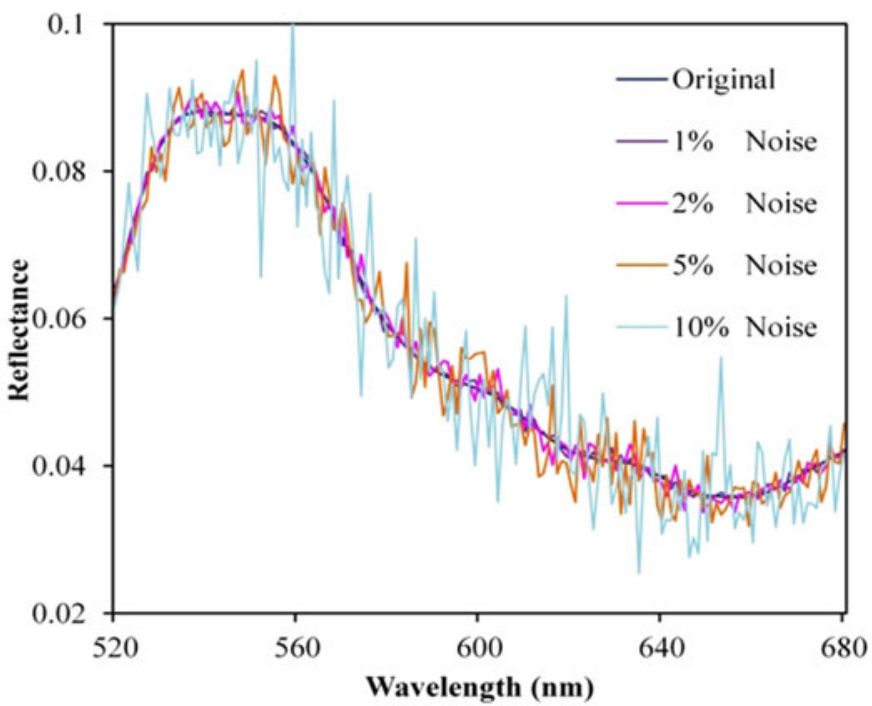

(d)

Fig. 2. Demonstration of spectra corresponding to bioparameter variation and noise interference. (a)-(c) represent spectra corresponding to variations of Chl, Car, and LWC, respectively; (d) illustrates the spectra with different levels of noise interference within 520-680 nm.

but also strong noise-resistant capability. To indicate noiseresistant capability of both WFs and CFs, Fig. 5 demonstrates the variation curves of retrieving accuracy (i.e., RMSE) under different noise levels. One can assess the noise-resistant capability of a feature through the slope of the curve. The flat curve indicates strong noise resistance whereas the steep slope indicates obvious influence of noise. In addition, the DecayRate provides a quantitative way to reflect feature's noise-resistant capability.

The WFs responded differently to leaf chemical parameters with respect to features' scales. It is noticeable that the highscale WFs tend to have relatively strong noise-resistant capability. Such a pattern embodied the nature of the wavelet analysis as a convolutional filter performed on the spectral data. If we treat the wavelet transformation as a band pass filter, the CWA will act as a traversal selection mechanism on band (i.e., central wavelength) and pass frequency (i.e., scale factor). All models for retrieving both $\mathrm{Chl}$ and LWC that were constructed by low-scale WFs (scale $\in[2,5]$ ) were significantly affected by noise, with DecayRate greater than 0.2. Two high-scale WFs (scale $=6$ ) corresponding to Car retrieval exhibited strong resistance to noise, with DecayRate lower than 0.1. Therefore, in our case, the scale $=6$ could be a cut point for identifying noise-resistant WFs in retrieving Chl, Car, and LWC. Besides, it should be noted that the locations of high-scale WFs for pigments differed from those for water content. The high-scale WFs for both $\mathrm{Chl}$ and Car distributed around red-edge and NIR regions (700-860 nm), whereas the high-scale WFs for LWC distributed around NIR and SWIR regions (1200-1350 and 1700-1900 nm). While for CFs, it was surprising that most VIs were seldom affected by noise in retrieving leaf chemical parameters, despite the VIs produced much lower 

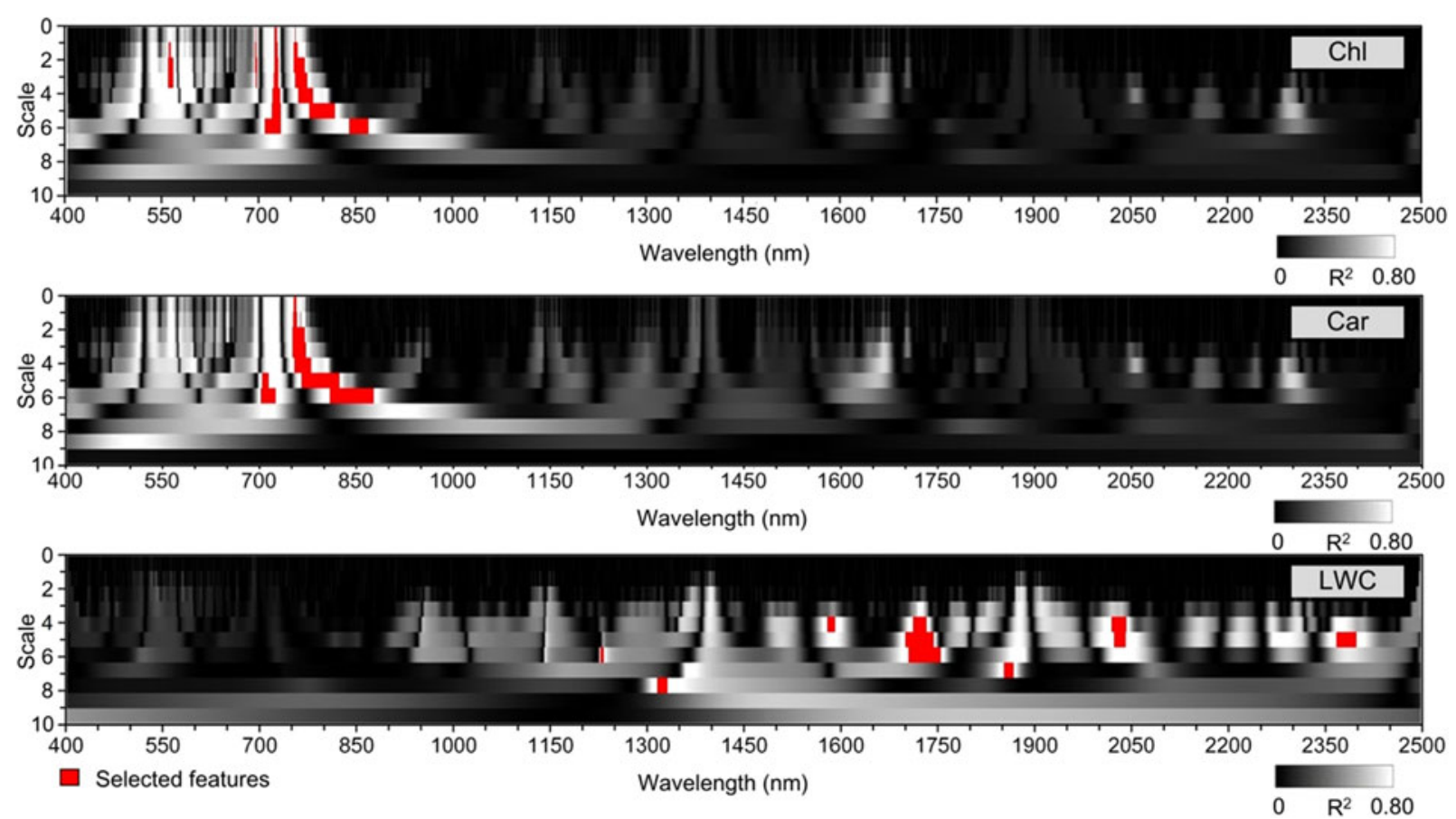

Fig. 3. Wavelet feature regions identified from correlation scalograms. The correlation scalograms for Chl, Car, and LWC are demonstrated with the selected feature regions marked in red color.

TABLE IV

Correlations Between Leaf Chemical Parameters And WaVelet Features/Conventional Features

\begin{tabular}{|c|c|c|c|c|c|c|c|c|c|c|c|c|}
\hline \multirow[b]{2}{*}{ Category } & \multicolumn{4}{|c|}{ Chl } & \multicolumn{4}{|c|}{ Car } & \multicolumn{4}{|c|}{ LWC } \\
\hline & Features & Scale & $\mathrm{CW}$ & $R^{2}$ & Features & Scale & $\mathrm{CW}$ & $R^{2}$ & Features & Scale & $\mathrm{CW}$ & $R^{2}$ \\
\hline \multirow[t]{7}{*}{ Wavelet features } & WF1 & 2 & 559 & 0.79 & $\underline{\mathrm{WF} 1}$ & 6 & 709 & 0.64 & WF1 & 6 & 1229 & 0.86 \\
\hline & WF2 & 2 & 693 & 0.79 & $\overline{\mathrm{WF} 2}$ & 6 & 852 & 0.66 & WF2 & 8 & 1322 & 0.73 \\
\hline & $\underline{W F} 3$ & 6 & 717 & 0.82 & & & & & $\overline{\mathrm{WF} 3}$ & 4 & 1585 & 0.74 \\
\hline & WF4 & 3 & 764 & 0.82 & & & & & WF4 & 6 & 1737 & 0.85 \\
\hline & $\underline{\text { WF5 }}$ & 6 & 855 & 0.79 & & & & & WF5 & 7 & 1861 & 0.80 \\
\hline & & & & & & & & & WF6 & 4 & 2036 & 0.76 \\
\hline & & & & & & & & & WF7 & 5 & 2385 & 0.76 \\
\hline \multirow[t]{5}{*}{ VIs } & $\underline{\mathrm{mND} 705}$ & & & 0.65 & PSSR & & & 0.03 & LWVI-1 & & & 0.22 \\
\hline & $\overline{\text { TVI }}$ & & & 0.35 & CRI550 & & & 0.00 & LWVI-2 & & & 0.34 \\
\hline & MCARI & & & 0.34 & CRI700 & & & 0.00 & MSI & & & 0.40 \\
\hline & mND680 & & & 0.06 & $\underline{\mathrm{DD}}$ & & & 0.56 & NDWI & & & 0.40 \\
\hline & $\underline{\text { CARI }}$ & & & 0.51 & & & & & $\overline{\mathrm{WI}}$ & & & 0.45 \\
\hline \multirow[t]{9}{*}{ Der \& Con features } & DEP-1 & & & 0.22 & DEP-1 & & & 0.09 & DEP-2 & & & 0.25 \\
\hline & AREA-1 & & & 0.02 & AREA-1 & & & 0.00 & AREA-2 & & & 0.32 \\
\hline & WID-1 & & & 0.43 & WID-1 & & & 0.34 & WID-2 & & & 0.00 \\
\hline & $\mathrm{Db}$ & & & 0.72 & $\mathrm{Db}$ & & & 0.48 & DEP-3 & & & 0.26 \\
\hline & $\underline{S D b}$ & & & 0.65 & $\mathrm{SDb}$ & & & 0.42 & AREA-3 & & & 0.27 \\
\hline & $\overline{D y}$ & & & 0.52 & Dy & & & 0.40 & WID-3 & & & 0.02 \\
\hline & SDy & & & 0.68 & SDy & & & 0.46 & & & & \\
\hline & $\overline{\mathrm{Dr}}$ & & & 0.03 & $\overline{\mathrm{Dr}}$ & & & 0.07 & & & & \\
\hline & $\mathrm{SDr}$ & & & 0.01 & SDr & & & 0.00 & & & & \\
\hline
\end{tabular}

The underlined features are selected for establishing the retrieving models; $\mathrm{CW}$ indicates central wavelength (nm).

accuracies than WFs (see Fig. 5). The only exception was the WI, which produced a DecayRate of 0.36. Unlike the VIs, the Der \& Con features had varied noise sensitivity. In retrieving Chl and Car, Db, Dy, and Dy showed strong response to noise, while in retrieving LWC, DEP-2, AREA-2, and AREA-3 responded strongly to noise. Besides, the other Der \& Con features performed relatively stable under noise interference. The retrieving accuracies of the Der \& Con features were also significantly lower than WFs. Such results suggested that many WFs and CFs have considerable noise-resistant capability, which were suitable for establishing retrieving models of leaf chemical parameters. 


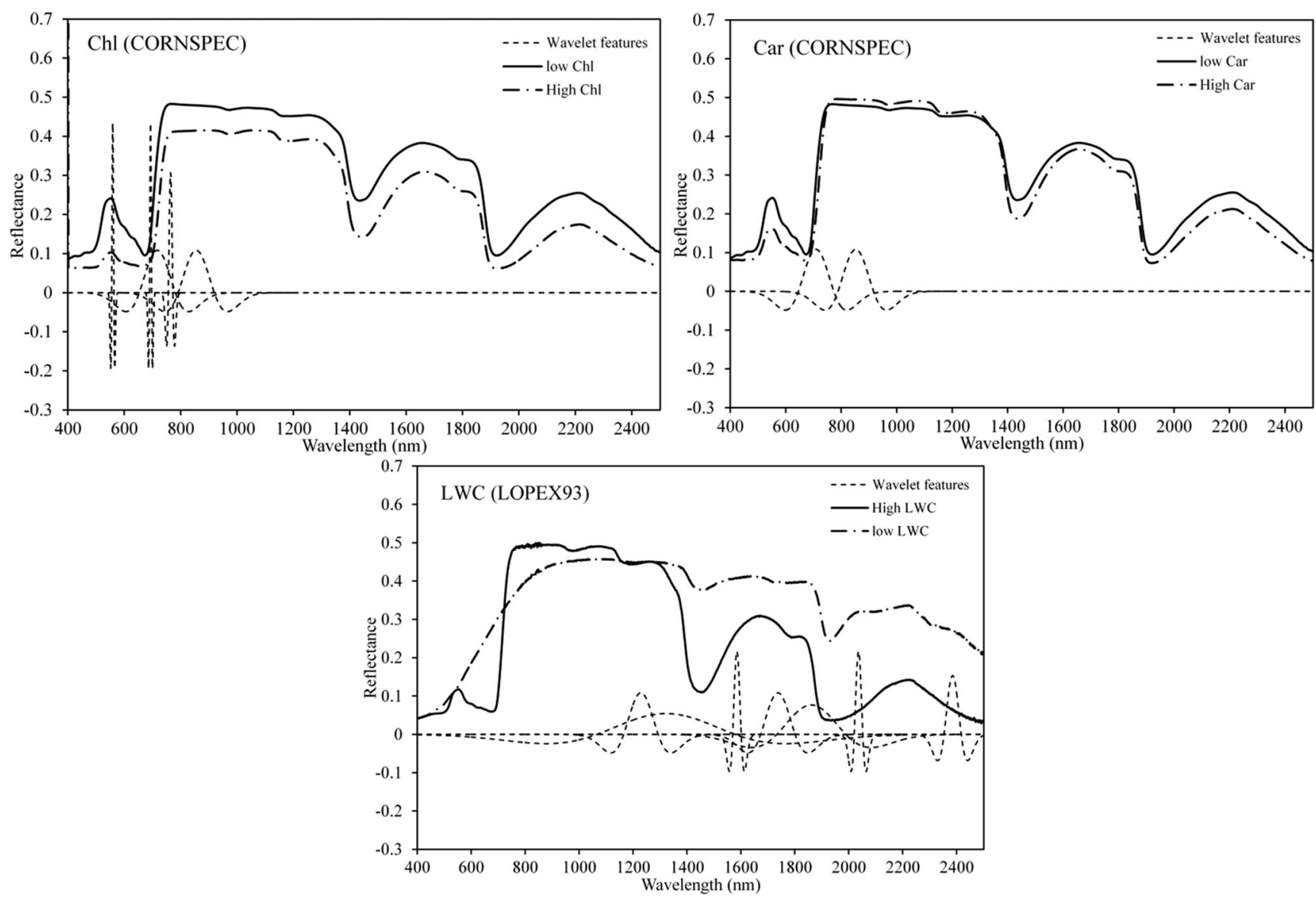

Fig. 4. Reference reflectance spectra indicating leaf chemical parameters variations (two spectra indicating low and high values for each parameter) and corresponding wavelet features (dash line indicating scale and central wavelength location).

\section{Retrieving Models of Leaf Chemical Parameters}

Using the identified WFs and CFs as input variables, the retrieving models for the three leaf chemical parameters were established with both the univariate and multivariate analysis. According to the results of features' sensitivity analysis and noise resistance assessment, features satisfying not only statistical significant correlation $(p$-value $<0.01$ ) with corresponding leaf chemical parameter but also the DecayRate lower than 0.2 were retained as input variables of retrieving models. Based on these features, both univariate and multivariate models were established for retrieving each leaf chemical parameter. The retrieving accuracies of the models were validated against both noise free data and data with $10 \%$ noise (see Table V).

The WF-models outperformed the CF-models significantly for all leaf chemical parameters, which was consistent with the pattern as revealed by features' sensitivity analysis. Between the two pigments, WF-model for $\mathrm{Chl}$ produced a remarkably higher retrieving accuracy $\left(R^{2}=0.83\right)$ than the WF-model for Car $\left(R^{2}=0.66\right)$. For LWC, a relatively high retrieving accuracy could be achieved by WF-model, with $R^{2}=0.87$. Comparing with the retrieving accuracies between models established by original data and data with $10 \%$ noise (see Table V), it was encouraging that only tiny difference was found. Such a result suggested that the retrieving model would not be affected by normal intensity noise. As for the performance between univariate models and multivariate models, the multivariate models produced equal or slightly higher accuracies than univariate models. However, the difference between them was insignificant.

\section{DisCUSSIONS}

All the WFs that were identified as most sensitive to leaf chemical parameters reflected the critical spectral absorption traits for corresponding parameters. For Chl and Car, many WFs located around green peak (centered at $559 \mathrm{~nm}$ ), red valley (centered at $693 \mathrm{~nm}$ ), and red edge (centered at 709, 717, $764 \mathrm{~nm}$ ), which were all important positions in VIS-NIR spectral region. Similarly, the identified WFs for LWC also located around $1300 \mathrm{~nm}$, where there is the absorption region of foliar water as mentioned by Curran [3]. Besides, these positions were also reported by Cheng et al. [17] in retrieving LWC with WFs. To process the wavelet analysis, the best capturing of changes on shape of spectral curves can be achieved by compressing and expanding the wavelet (change of scale factor), or shifting its position (change of central wavelength). Given the shape of Mexican Hat wavelet fitted well with the absorption, reflection, and scattering features of the vegetation spectra, the WFs performed well in characterizing spectral responses of the leaf biochemical 


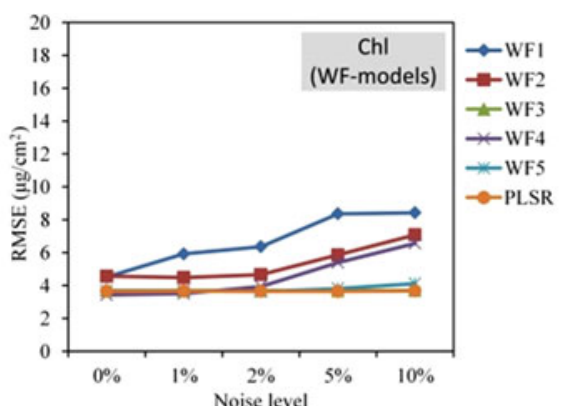

(a)

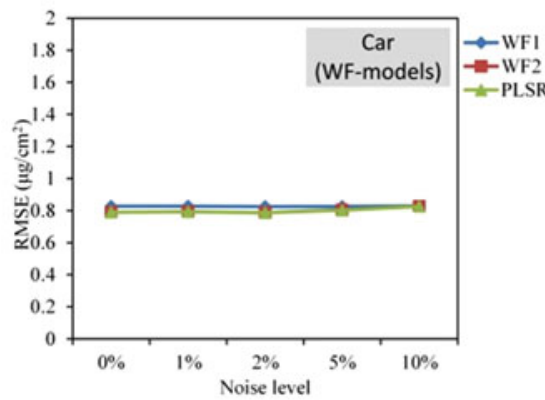

(d)

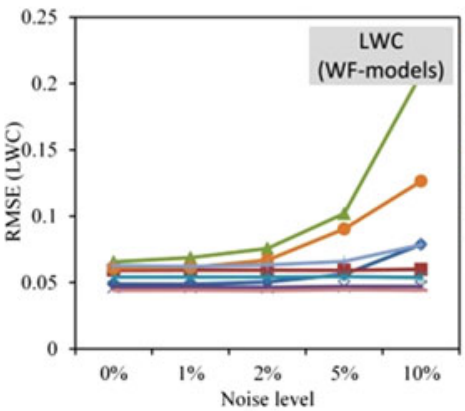

(g)

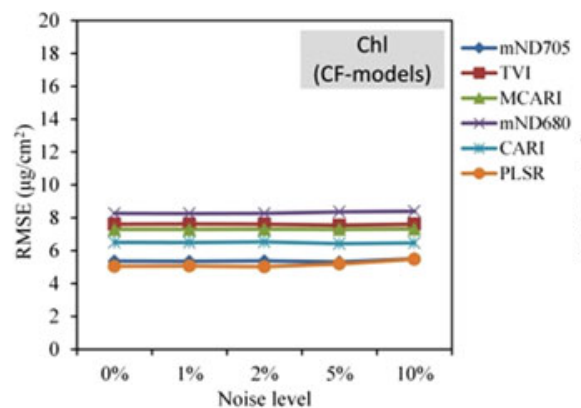

(b)

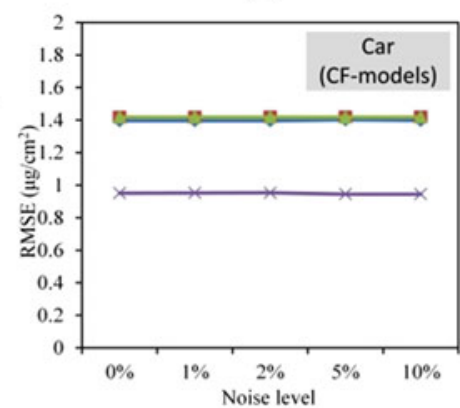

(e)

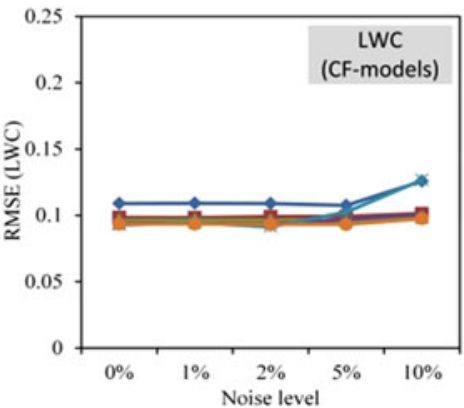

(h)

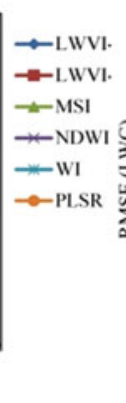

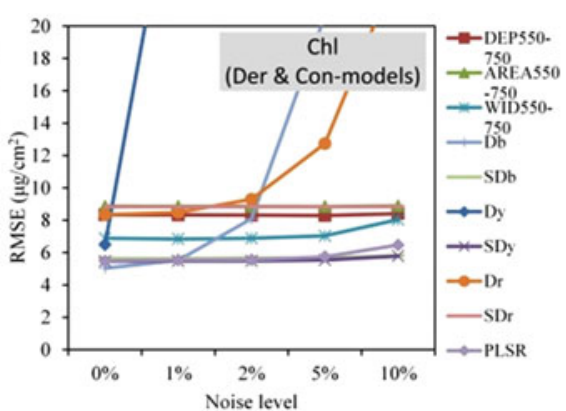

(c)

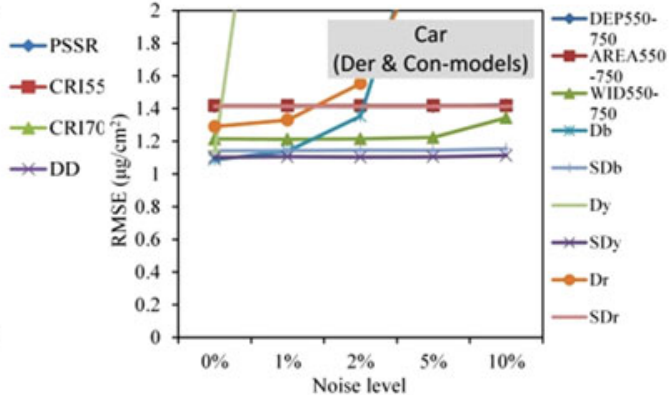

(f)

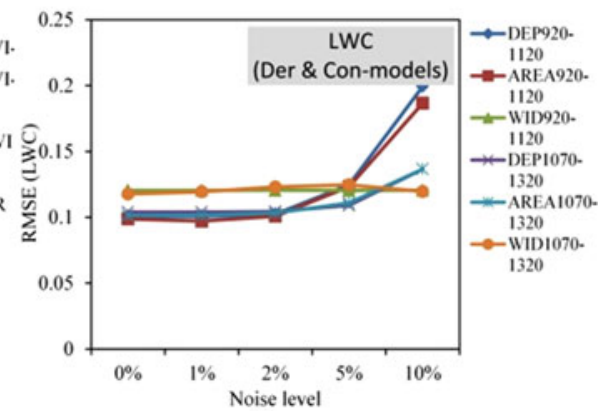

(i)

Fig. 5. Noise sensitivity of different types of models. The noise sensitivity is indicated by RMSE under different noise levels. The model types including WF-models, CF-models, and Der \& Con-models are compared. (a-c) models for retrieving Chl; (d-f) models for retrieving Car; and (g-i) for retrieving LWC.

parameters (see Fig. 4). It should be noted that in most cases, the shape-based spectral features would be more robust than spectral features based on bands' magnitude (e.g., some VIs). While comparing with some other shape-based spectral features, such as Der \& Con features that are also applied in this study, no spectral normalization is required for WFs, which makes them more robust and superior in dealing with background interferences (i.e., differences in illumination for measurement) [33]. These interior advantages of WFs thus accounted for the notable higher sensitivity to leaf chemical parameters comparing with CFs.

Both WFs and some CFs exhibited strong noise-resistant capability in retrieving leaf chemical parameters. For WFs, a clear relationship was observed between features' scale factor and their noise-resistant capabilities, where high-scale WFs tend to have strong noise-resistant capability. Given that the high-scale WFs tend to capture global spectral variation over broad regions, they are less sensitive to high-frequency noise. Such trait of high-scale WFs permits the analysis based on original spectra directly, which requires no spectral filtering procedure and thus avoids any possible information loss. Together with the sensitivity analysis, a number of high-scale WFs were identified for retrieving Chl (WF3, WF5), Car (WF1, WF2), and LWC (WF1, WF2, WF4, WF5) (see Table IV), which thus ensured the noise resistivity of WF-models. Despite the evident reason for the lack of strong noise-resistant capability of VIs, some possible explanations were provided. On the one hand, the arithmetic terms of VIs (i.e., normalization, ratio, etc.) might play a role in mitigating the noise impact in retrieving leaf chemical parameters. On the other hand, the relatively weak sensitivity of VIs to leaf chemical parameters (see Table IV) would cause a considerable uncertainty in retrieving, which might also weaken the influence of noise [37]. While for the Der \& Con features, despite most features had strong noise resistivity, some of them responded sensitively to the noise. For example, given the spectral derivative processing would enlarge the difference of neighboring bands, the maximum value of first derivative within blue edge (Db), yellow edge (Dy), and red edge (Dr) appeared to be susceptible to noise. While given the sum of first derivative values within the spectral edge regions would offset 
TABLE V

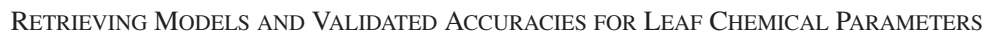

\begin{tabular}{|c|c|c|c|c|c|c|c|}
\hline \multirow{3}{*}{ Leaf chemical parameters } & \multirow{3}{*}{ Input variables } & \multirow{3}{*}{ Model equations } & \multirow{3}{*}{ Decay rate } & \multicolumn{4}{|c|}{ Validation accuracies } \\
\hline & & & & \multicolumn{2}{|c|}{ Noise free } & \multicolumn{2}{|c|}{$10 \%$ Noise } \\
\hline & & & & $R^{2}$ & RMSE & $R^{2}$ & RMSE \\
\hline \multirow[t]{3}{*}{ Chl (WF models) } & WF3 & $\mathrm{Chl}=-105.69 * \mathrm{WF} 3+31.07$ & $0.27 \%$ & 0.83 & 3.69 & 0.83 & 3.70 \\
\hline & WF5 & $\mathrm{Chl}=556.47 * \mathrm{WF} 5-22.80$ & $12.31 \%$ & 0.83 & 3.66 & 0.78 & 4.11 \\
\hline & PLSR & $\mathrm{Chl}=-98.70 * \mathrm{WF} 3+39.05 * \mathrm{WF} 5+27.21$ & $0.63 \%$ & 0.83 & 3.65 & 0.83 & 3.67 \\
\hline \multirow[t]{5}{*}{ Chl (VI models) } & mND705 & $\mathrm{Chl}=117.23 * \mathrm{mND} 705-21.28$ & $2.76 \%$ & 0.63 & 5.36 & 0.61 & 5.51 \\
\hline & TVI & $\mathrm{Chl}=-3.46 * \mathrm{TVI}+138.26$ & $0.14 \%$ & 0.26 & 7.61 & 0.25 & 7.62 \\
\hline & MCARI & $\mathrm{Chl}=-821.82 * \mathrm{MCARI}+77.49$ & $0.32 \%$ & 0.32 & 7.29 & 0.31 & 7.32 \\
\hline & CARI & $\mathrm{Chl}=-80.87 * \mathrm{CARI}+80.28$ & $0.40 \%$ & 0.46 & 6.50 & 0.46 & 6.47 \\
\hline & PLSR & $\mathrm{Chl}=-55.28+155.99 * \mathrm{mND} 705-0.31 * \mathrm{TVI}+739.25 * \mathrm{MCARI}-17.50 * \mathrm{CARI}$ & $8.45 \%$ & 0.67 & 5.05 & 0.62 & 5.48 \\
\hline \multirow[t]{4}{*}{ Chl (Der \& Con model) } & WID550-750 & $\mathrm{Chl}=2.23 *$ WID1 -196.92 & $16.79 \%$ & 0.39 & 6.88 & 0.25 & 8.04 \\
\hline & $\mathrm{SDb}$ & $\mathrm{Chl}=-421.86 * \mathrm{SDb}+79.65$ & $3.06 \%$ & 0.59 & 5.64 & 0.57 & 5.81 \\
\hline & SDy & $\mathrm{Chl}=720.83 * \mathrm{SDy}+82.20$ & $5.98 \%$ & 0.62 & 5.46 & 0.57 & 5.78 \\
\hline & PLSR & $\mathrm{Chl}=55.17+0.24 * \mathrm{WID} 1+435.93 * \mathrm{SDb}+1394.27 * \mathrm{SDy}$ & $18.17 \%$ & 0.62 & 5.47 & 0.49 & 6.47 \\
\hline \multirow[t]{3}{*}{ Car (WF models) } & WF1 & $\mathrm{Car}=-16.78 * \mathrm{WF} 1+0.47$ & $0.26 \%$ & 0.66 & 0.83 & 0.66 & 0.83 \\
\hline & WF2 & $\mathrm{Car}=70.79 * \mathrm{WF} 2-1.46$ & $4.62 \%$ & 0.70 & 0.79 & 0.67 & 0.83 \\
\hline & PLSR & $\mathrm{Car}=-1.50+0.40 * \mathrm{WF} 1+72.45 * \mathrm{WF} 2$ & $4.64 \%$ & 0.70 & 0.79 & 0.66 & 0.83 \\
\hline Car (VI model) & $\mathrm{DD}$ & $\mathrm{Car}=33.11 * \mathrm{DD}+5.43$ & $0.22 \%$ & 0.55 & 0.95 & 0.57 & 0.94 \\
\hline Car (Der \& Con model) & SDy & $\mathrm{Car}=91.81 * \mathrm{SDy}+13.11$ & $1.00 \%$ & 0.40 & 1.10 & 0.38 & 1.11 \\
\hline \multirow[t]{4}{*}{ LWC (WF models) } & WF2 & $\mathrm{LWC}=-0.89 * \mathrm{WF} 2+0.65$ & $1.10 \%$ & 0.77 & 0.059 & 0.76 & 0.060 \\
\hline & WF4 & $\mathrm{LWC}=3.02 * \mathrm{WF} 4+0.49$ & $1.41 \%$ & 0.86 & 0.046 & 0.85 & 0.047 \\
\hline & WF5 & $\mathrm{LWC}=-1.88 * \mathrm{WF} 5+0.56$ & $0.80 \%$ & 0.80 & 0.054 & 0.80 & 0.054 \\
\hline & PLSR & $\mathrm{LWC}=0.51-0.05 * \mathrm{WF} 2+2.21 * \mathrm{WF} 4-0.47 * \mathrm{WF} 5$ & $1.14 \%$ & 0.87 & 0.044 & 0.87 & 0.044 \\
\hline \multirow[t]{4}{*}{ LWC (VI models) } & LWVI-2 & $\mathrm{LWC}=4.78 * \mathrm{LWVI}-2+0.50$ & $2.91 \%$ & 0.33 & 0.098 & 0.31 & 0.101 \\
\hline & MSI & $\mathrm{LWC}=-0.79 * \mathrm{MSI}+1.19$ & $2.03 \%$ & 0.36 & 0.096 & 0.34 & 0.098 \\
\hline & NDWI & $\mathrm{LWC}=3.97 * \mathrm{NDWI}+0.54$ & $6.98 \%$ & 0.39 & 0.094 & 0.31 & 0.101 \\
\hline & PLSR & $\mathrm{LWC}=0.96-0.79 * \mathrm{LWVI}-2-0.51 * \mathrm{MSI}+2.22 * \mathrm{NDWI}$ & $4.30 \%$ & 0.40 & 0.093 & 0.35 & 0.098 \\
\hline
\end{tabular}

the neighboring noise effects, the SDb, SDy, and SDr were less sensitive to the noise. Such phenomenon was also observed by Cloutis [38].

Benefited from the capability of CWA in characterizing spectral response due to variation of leaf chemical parameters and the strong noise resistivity of high-scale WFs, the WF-models outperformed the VI-models or Der \& Con-models in accuracy and robustness in retrieving Chl, Car, and LWC (see Table V). While for preference of model forms, considering the multivariate models tend to significantly increase the complexity in features' calculation yet lead to unobvious improvement in accuracy (see Table V), they are not recommended in this study. Conversely, given that the optimal univariate WF-models are able to achieve similar level of retrieving accuracy under a noise interference circumstances, they are recommended in practical applications.

It is worth noting that the WFs and WF-models have a potential to be utilized for retrieving the biochemical parameters at field scale or regional scale with the aid of airborne or satellite remote sensing images. As the wavelet analysis is performed on the entire vegetation spectrum, the capability in acquiring hyperspectral image data is mandatory for the corresponding sensors. It is encouraging that the miniaturized lightweight hyperspectral imaging spectrometers (e.g., UHD185, hyperspectral UAV for UAV platform) and hyperspectral satellite sensors (e.g., HyspIRI, PRISMA) are undergoing a rapid development. To examine the feasibility of the WF-models in retrieving biochemical parameters with these airborne/satellite hyperspectral image data, some field experiments are necessary. Moreover, to find whether the spectral resolution of the airborne/satellite hyperspectral sensors is adequate for processing the CWA analysis, more efforts are expected for understanding the influence of spectral continuity on feature/model's performance in retrieving different leaf chemical parameters.

\section{CONCLUSION}

In retrieving some critical leaf chemical parameters including Chl, Car, and LWC, the WFs that were derived from CWA significantly outperformed the CFs that consisted VIs, derivative, and continuum-removal features. Both forms of features exhibited strong resistant capability to up to $10 \%$ spectral noise. The high-scale WFs tend to have strong resistivity to noise. Through an independent validation dataset, the best models achieved $R^{2}$ of $0.83,0.66$, and 0.87 in retrieving Chl, Car, and LWC, respectively. As a tradeoff between models' performance and complexity, the univariate WF-models were recommended for retrieving those leaf chemical parameters in practice.

\section{ACKNOWLEDGMENT}

The authors are grateful to W. Li and H. Chang for their help in field data collection. 


\section{REFERENCES}

[1] F. Bussotti, M. Pollastrini, and F. Bussotti, "Evaluation of leaf features in forest trees: Methods, techniques, obtainable information and limits," Ecol. Indicators, vol. 52, pp. 219-230, 2015.

[2] G. P. Asner and R. E. Martin, "Spectral and chemical analysis of tropical forests: Scaling from leaf to canopy levels," Remote Sens. Environ., vol. 112, pp. 3958-3970, 2008.

[3] P. J. Curran, "Remote sensing of foliar chemistry," Remote Sens. Environ., vol. 30, pp. 271-278, 1989.

[4] S. L. Ustin et al., "Retrieval of foliar information about plant pigment systems from high resolution spectroscopy," Remote Sens. Environ., vol. 1, pp. S67-S77, 2009.

[5] M. S. Kim, C. S. T. Daughtry, E. W. Chappelle, and J. E. McMurtrey, "The use of high spectral resolution bands for estimating absorbed photosynthetically active radiation (APAR)," in Proc. 6th Int. Symp. Phys. Meas. Signatures Remote Sens., vol. 8, Val d'Isere, France, 1994, pp. 299-306.

[6] A. A. Gitelson, Y. Zur, O. B. Chivkunova, and M. N. Merzlyak, "Assessing carotenoid content in plant leaves with reflectance spectroscopy," Photochem. Photobiol., vol. 75, pp. 272-281, 2002.

[7] A. A. Gitelson, G. P. Keydan, and M. N. Merzlyak, "Three-band model for noninvasive estimation of chlorophyll, carotenoids, and anthocyanin contents in higher plant leaves," Geophys. Res. Lett., vol. 33, pp. 431-433, 2006

[8] C. S. T. Daughtry, C. L. Walthall, M. S. Kim, E. B. D. Colstoun, and M. M. Iii, "Estimating corn leaf chlorophyll concentration from leaf and canopy reflectance," Remote Sens. Environ., vol. 74, pp. 229-239, 2000.

[9] A. A. Gitelson, Y. Peng, T. J. Arkebauer, and J. Schepers, "Relationships between gross primary production, green LAI, and canopy chlorophyll content in maize: Implications for remote sensing of primary production," Remote Sens. Environ., vol. 144, pp. 65-72, 2014.

[10] E. R. Hunt and B. N. Rock, "Detection of changes in leaf water content using Near-and Middle-Infrared reflectances," Remote Sens. Environ., vol. 30, pp. 43-54, 1989.

[11] J. Penuelas, J. Pinol, R. Ogaya, and I. Filella, "Estimation of plant water concentration by the reflectance water index WI (R900/R970)," Int. J. Remote Sens., vol. 18, pp. 2869-2875, 1997.

[12] Q. H. Weng, "Advances in environmental remote sensing," in Hyperspectral Remote Sensing of Vegetation Bioparameters, R. L. Pu and P. Gong, Editors. Boca Raton, FL, USA: CRC Press, 2011, ch. 5.

[13] R. N. Clark and T. L. Roush, "Reflectance spectroscopy: quantitative analysis techniques for remote sensing applications," J. Geophys. Res., vol. 89, pp. 6329-6340, 1984.

[14] R. Pu, S. Ge, N. M. Kelly, and P. Gong, "Spectral absorption features as indicators of water status in Quercus agrifolia leaves," Int. J. Remote Sens., vol. 24, pp. 1799-1810, 2003.

[15] G. A. Blackburn, "Wavelet decomposition of hyperspectral data: A novel approach to quantifying pigment concentrations in vegetation," Int. J. Remote Sens., vol. 28, pp. 2831-2855, 2007.

[16] G. A. Blackburn and J. G. Ferwerda, "Retrieval of chlorophyll concentration from leaf reflectance spectra using wavelet analysis," Remote Sens. Environ., vol. 112, pp. 1614-1632, 2008.

[17] T. Cheng, B. Rivard, A. G. Sánchez-Azofeifa, J. Féret, S. Jacquemoud, and S. L. Ustin, "Predicting leaf gravimetric water content from foliar reflectance across a range of plant species using continuous wavelet analysis," J. Plant Physiol., vol. 169, pp. 1134-1142, 2012.

[18] T. Cheng, B. Rivard, A. G. Sánchez-Azofeifa, J. B. Féret, S. Jacquemoud, and S. L. Ustin, "Deriving leaf mass per area (LMA) from foliar reflectance across a variety of plant species using continuous wavelet analysis," Int. J. Photogram. Remote Sens., vol. 87, pp. 28-38, 2014.

[19] T. Cheng, D. Riaño, A. Koltunov, M. L. Whiting, S. L. Ustin, and J. Rodriguez, "Detection of diurnal variation in orchard canopy water content using MODIS/ASTER airborne simulator (MASTER) data," Remote Sens. Environ., vol. 132, pp. 1-12, 2013.

[20] T. Cheng, D. Riaño, and S. L. Ustin, "Detecting diurnal and seasonal variation in canopy water content of nut tree orchards from airborne imaging spectroscopy data using continuous wavelet analysis," Remote Sens. Environ., vol. 143, pp. 39-53, 2014.

[21] J. Luo, W. Huang, J. Zhao, J. Zhang, C. Zhao, and R. Ma, "Detecting aphid density of winter wheat leaf using hyperspectral measurement," IEEE J. Sel. Topics Appl. Earth Obs. Remote Sens., vol. 6, pp. 690-698, 2013.

[22] H. F. Wang, Z. G. Huo, G. S. Zhou, Q. H. Liao, H. K. Feng, and L. $\mathrm{Wu}$, "Estimating leaf SPAD values of freeze-damaged winter wheat using continuous wavelet analysis," Plant Physiol. Biochem., vol. 98, pp. 39-45, 2015.
[23] J. Zhang, R. Pu, R. W. Loraamm, G. Yang, and J. Wang, "Comparison between wavelet spectral features and conventional spectral features in detecting yellow rust for winter wheat," Comput. Electron. Agric., vol. 100 pp. 79-87, 2014.

[24] I. Levin, E. Levina, G. Gilbert, and S. Stewart, "Role of sensor noise in hyperspectral remote sensing of natural waters: Application to retrieval of phytoplankton pigments," Remote Sens. Environ., vol. 95, pp. 264-271, 2005.

[25] A. Majumdar, N. Ansari, H. Aggarwal, and P. Biyani, "Impulse denoising for hyper-spectral images: A blind compressed sensing approach," Signal Process., vol. 119, pp. 136-141, 2016.

[26] H. K. Lichtenthaler, "Chlorophylls and carotenoids: pigments of photosynthetic biomembranes," Methods Enzymol., vol. 148, pp. 350-382, 1987.

[27] B. Hosgood, S. Jacquemoud, G. Andreoli, J. Verdebout, G. Pedrini, and G. Schmuck, "Leaf optical properties experiment 93 (LOPEX93)," Eur Commission, Joint Res. Centre, Inst. Remote Sens. Appl., Italy, Rep. no. EUR 16095 EN, 1995.

[28] L. Li, S. L. Ustin, and D. Riano, "Retrieval of fresh leaf fuel moisture content using genetic algorithm partial least squares (GA-PLS) modeling," IEEE Geosci. Remote Sens. Lett., vol. 4, no. 2, pp. 216-220, Apr. 2007.

[29] D. Riano, P. Vaughan, E. Chuvieco, P. J. Zarco-Tejada, and S. L. Ustin, "Estimation of fuel moisture content by inversion of radiative transfer models to simulate equivalent water thickness and dry matter content: Analysis at leaf and canopy level," IEEE Trans. Geosci. Remote Sens., vol. 43, no. 4, pp. 819-826, Apr. 2005.

[30] L. M. Bruce, J. Li, and Y. Huang, "Automated detection of subpixel hyperspectral targets with adaptive multichannel discrete wavelet transform," IEEE Trans. Geosci. Remote Sens., vol. 40, no. 4, pp. 977-980, Apr. 2002.

[31] L. Ebadi and H. Z. M. Shafri, "A stable and accurate wavelet-based method for noise reduction from hyperspectral vegetation spectrum," Earth Sci. Informat., vol. 8, pp. 411-425, 2014.

[32] S. Kaewpijit, J. Le Moigne, and T. El-Ghazawi, "Automatic reduction of hyperspectral imagery using wavelet spectral analysis," IEEE Trans. Geosci. Remote Sens., vol. 41, no. 4, pp. 863-871, Apr. 2003.

[33] T. Cheng, B. Rivard, and A. Sanchez-Azofeifa, "Spectroscopic determination of leaf water content using continuous wavelet analysis," Remote Sens. Environ., vol. 115, pp. 659-670, 2011.

[34] L. M. Bruce and J. Li, "Wavelets for computationally efficient hyperspectral derivative analysis," IEEE Trans. Geosci. Remote Sens., vol. 39, no. 7, pp. 1540-1546, Jul. 2001

[35] C. Torrence and G. P. Compo, "A practical guide to wavelet analysis," Bull. Amer. Meteorol. Soc., vol. 79, pp. 61-78, 2010.

[36] Y. Wang and J. Y. Mo, "A new de-noising technique for spectra based on Mexican hat wavelet," Spectrosc. Spectr. Anal., vol. 25, pp. 124-127, 2005.

[37] L. Zhang, L. Zhang, L. Yan, N. Fujiwara, K. Muramatsu, and M. Daigo, "Hyperspectral data transformation and vegetation index performance based on the universal pattern decomposition method," J. Imag. Sci. Technol., vol. 51, pp. 4919-4928, 2007.

[38] E. A. Cloutis, "Hyperspectral geological remote sensing: Evaluation of analytical techniques," Int. J. Remote Sens., vol. 17, pp. 2215-2242, 1996.

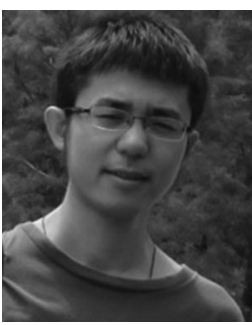

Jingcheng Zhang received the Ph.D. degree in agricultural remote sensing and information technology from Zhejiang University, Hangzhou, China, in 2012.

$\mathrm{He}$ is currently an Associate Professor in the College of Life Information Science and Instrument Engineering, Hangzhou Dianzi University, Hangzhou, China. His current research interests include spectral analysis on crop growing status, remotely sensed detection and monitoring of crop diseases and pests, and forecasting of crop diseases and pests by integrating weather data and RS data. 


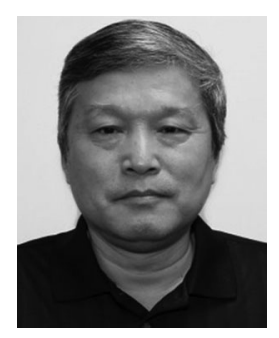

Yanbo Huang received the Ph.D. degree in agricultural engineering from Texas A\&M University, College Station, TX, USA, in 1995.

Currently, he is a Senior Research Agricultural Engineer and the Lead Scientist in the Program of Aerial Application Technology and Remote Sensing in United Department of Agriculture, Agricultural Research Service, Crop Production Systems Research Unit, Stoneville, MS, USA. His current research interests include agricultural remote sensing and data mining techniques.

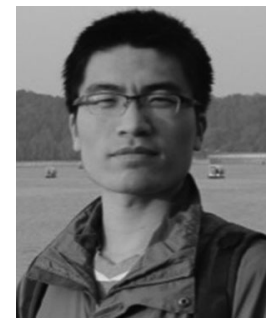

Zhenhai Li received the Ph.D. degree in agricultural remote sensing and information technology from Zhejiang University, Hangzhou, China, in 2016.

$\mathrm{He}$ is currently an Assistant Professor in Beijing Research Center for Information Technology in Agriculture, Beijing Academy of Agriculture and Forestry Sciences, Beijing, China. His current research interests include remote sensing analysis and diagnosis of crop growth and nitrogen, and crop yield and quality forecasting by assimilating crop growth model and remote sensing.

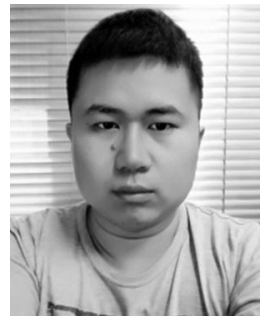

Peng Liu is working toward the Postgraduate degree in instruments science and technology at Hangzhou Dianzi University, Hangzhou, China.

His current research interests include agricultural disease detection and monitoring by hyperspectral remote sensing data.

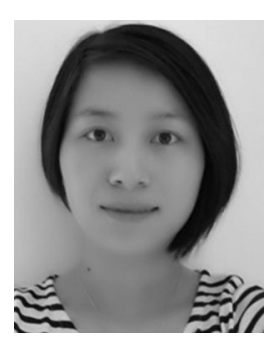

Lin Yuan received the Ph.D. degree in plant nutrition from Zhejiang University, Hangzhou, China, in 2015.

She is currently a Lecturer in the School of Information Engineering and Art and Design, Zhejiang University of Water Resources and Electric Power, Hangzhou, China. Her research experience and interests include hyperspectral analyzing, agricultural disease detection, and monitoring by incorporating multisources of remote sensing data. 